\title{
Numerical Simulation and Experimental Study of Powder Flow Distribution in High Power Direct Diode Laser Cladding Process
}

\author{
S. Liu ${ }^{1} \cdot$ Y. Zhang $^{2} \cdot$ R. Kovacevic ${ }^{1}$
}

Accepted: 21 August 2015 / Published online: 7 October 2015

(C) Springer New York 2015

\begin{abstract}
High power direct diode laser (HPDDL) offers a rectangular laser beam with top-flat intensity distribution making it an ideal tool for wide-clad deposition. In the HPDDL-based cladding process, the powder is commonly fed laterally through a wide nozzle into the molten pool by means of a carrier gas. In order to successfully utilize this cladding technique, the powder feeding behavior needs to be carefully controlled. In this study, based on an investigation of commercial powder feeding nozzles, a 3D computational fluid dynamics (CFD) based gas-powder flow model was developed. The effects of the nozzle exit geometry, powder properties (particle size, density and shape), and powder feeding parameters (powder feeding rate and carrier-gas flow rate) on the characteristics of the powder flow were studied. A high-speed CCD camera was used to capture the powder flow characteristics such as the particle velocity and particle distribution. Deposition experiments are also performed to verify the predicted powder catchment efficiency and to check the clad geometry.
\end{abstract}

Keywords High power direct diode laser (HPDDL) · Gas-powder flow · Computational fluid dynamics (CFD)

\section{Introduction}

Laser cladding is a promising technique for surface quality enhancement. A metallic or ceramic layer can be added locally to the surface of a workpiece to improve its hardness, wear, or corrosion resistance. Several tracks can be overlapped to cover a

R. Kovacevic

kovacevi@lyle.smu.edu

1 Center for Laser-Aided Manufacturing, Southern Methodist University, 3101 Dyer Street, Dallas, TX 75205, USA

2 Coherent Inc., 5100 Patrick Henry Drive, Santa Clara, CA 95054, USA 
large area, and the excess material can be removed by grinding or machining. Most of the previous research on laser cladding used $\mathrm{CO}_{2}$ and $\mathrm{Nd}$ :YAG lasers. The high power direct diode laser which is characterized by high electricity efficiency, a long lifetime, and low capital and operation costs, has found wide application in laser cladding [5]. With its short wavelength, HPDDL has an increase of beam absorption to metal with respect to $\mathrm{CO}_{2}$ and $\mathrm{Nd}: Y A G$ lasers [12]. Its wide laser beam with nearly uniform intensity distribution minimizes the dilution value as low as $0.2 \%$ [5] and is ideal for large area deposition.

Powder injection cladding is a one-step process that possesses the most versatility and highest accuracy for controlling the geometry of the clad and the deposition of the clad on complex surfaces. Powder can be fed laterally [8] or coaxially [3] into the laser beam. The major advantage of coaxial powder injection is its directional independence, which makes it suitable for the production of complex components. For large area cladding using a rectangular laser beam, the powder is usually fed laterally from a wide nozzle. The shape of the gas-powder stream, the interaction between powder stream and laser beam, and the molten pool play an important role in determining the clad geometry, clad quality, and powder catchment efficiency. Cladding processes require the understanding of numerous parameters, such as the nozzle geometry and its position, the beam shape with its intensity distribution, the carrier-gas flow rate, the powder feeding rate, the particle properties, and the substrate position. In-situ monitoring and numerical modeling were applied to improve the clad quality. Several online monitoring methods have been tried. Meriaudeau et al. [14] used one CCD camera to detect the temperature distribution of molten pool and another to detect the information related to the shape of the clad, in order to develop a closed-loop control to compensate the variation in the process. Doubenskaia et al. [6] used an infrared camera to visualize the thermal phenomena of the molten pool to get the information including the temperature distribution, thermal gradient, and cooling rate. $\mathrm{Hu}$ and Kovacevic [11] used a CCD camera to monitor the size of the molten pool to ensure the constant width of the deposition wall in laser additive manufacturing process. However, it is difficult for on-line monitoring of the cladding process due to the high intensity of the laser beam irradiating small area and while interacting with fine powder particles at a high speed. Hence, numerical modeling offers a cost-effective way to better understand the related physics in the laser cladding process and also provides guidance on optimizing the process parameters.

In the past decades, a significant amount of experimental, analytical and numerical modeling efforts have been dedicated to the development of cladding technology. Lin and Steen [13] were the first to carry out some work on the characteristics of the powder flow structure. They found that the coaxial powder stream was characterized by a Gaussian distribution. The powder catchment efficiency could be optimized by controlling the shielding- and carrier-gas flow rate. Pinkerton and Li $[17,18]$ proposed an analytical model to estimate the powder concentration distribution and its attenuation effect on laser beam. The powder flow visualization system was also developed to validate the predicted results. Zekovic et al. [25] built a computational fluid dynamics (CFD) model to simulate the gas-powder flow of coaxial powder injection. The developed model was used to estimate the optimal distance from the nozzle tip to the molten pool. Yang [24] found that the powder properties, nozzle geometry, and shielding gas had large influences on the powder concentration distribution. Balu 
et al. [3] modeled the powder flow behavior of mixing particles of Ni-WC. However, most of investigations so far have concentrated on the coaxial powder feeding system with a circular laser beam. A few reports studies on off-axis powder injection with respect to a rectangular laser beam. Wen et al.[23]) predicted the thermal behavior of the powder stream based on the assumption of uniform powder ejection from a rectangular outlet.

In order to make good use of the HDPPL cladding with off-axis powder injection, in this study, the effects of the nozzle geometry, particle properties (particle density, size, and shape), and powder feeding parameters on the characteristics of powder flow(including particle velocity, particle trajectory, and powder concentration distribution) were investigated by using FLUENT software. The numerical model was validated with the experimental results based on the powder flow images captured by a CCD camera and the deposited clad geometry.

\section{Experimental Investigation}

The experimental setup was comprised of an 8-kW Coherent HPDDL at a wavelength of $980 \pm 10 \mathrm{~nm}$, a 6-axis KUKA robot, and an AT-1200 high-pressure-rotary powder feeding system, and the laser head tilted equipped with a set of powder feeding nozzles, as shown in Fig. 1a. The HPDDL was focused into a $12 \times 3 \mathrm{~mm}$ rectangular spot. The divergence angle for the $\mathrm{x}$ (fast) axis and $\mathrm{z}$ (slow) axis were $\alpha=23^{\circ}$ and $\beta=19^{\circ}$, respectively. The powder flow ejected out from the nozzle was illuminated by a 532-nm wavelength green laser, as shown in Fig. 1b. The image was captured by a CCD camera at the exposure time of $0.02 \mathrm{~s}$. In order to reduce the effects of the random movement of particles, an average image was obtained from 25 continuous images. According to Mie theory (the scattering of light by small particles), the luminous intensity was proportional to the concentration of the powder flow. The particle velocity at the nozzle exit was obtained by a CCD camera at the exposure time of $0.0002 \mathrm{~s}$.

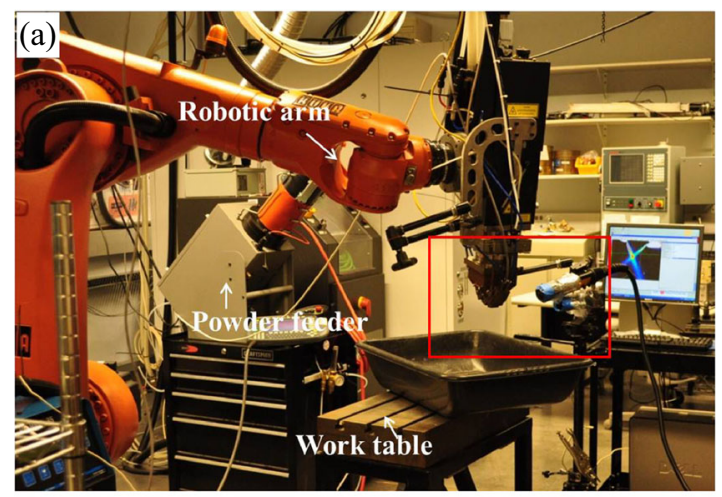

(b)

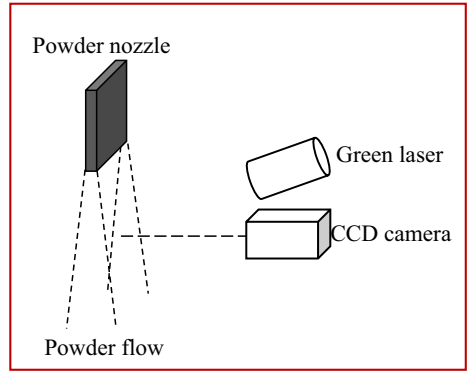

6-axis KUKA Robot-Controlled 8-kW Direct Diode Laser Manufacturing System

Fig. 1 (a) Experimental setup for laser cladding process and (b) vision system to study powder flow dynamic behavior 
Figure 2a schematically illustrates the configuration of the laser head installed with the powder-delivery nozzle. In order to protect the lens from the reflection of laser beam and the ricochet particles, the entire laser head was tilted $10^{\circ}$ with respect to the vertical axis. The position of the nozzle could be adjusted to obtain a good coupling between the powder stream and the diode laser beam. The powder was fed into the powder nozzle by the carrier gas. The existing nozzle design had an exit of seven distinct channels and as such was used for modeling as well as for the experiments. The channel was a square slot with the dimensions of $1 \times 1 \mathrm{~mm}$. The total exit area is $7 \mathrm{~mm}^{2}$. To investigate the effect of the nozzle exit geometry on the convergence of the powder flow, two types of nozzles (one with a closed exit shape and the other with an open exit shape) were tested, as shown in Fig. 2b-c.

Argon was used as carrier gas. An Fe-based alloy powder was used during the experiments, in which the particle size $D_{p}$ ranged from 45 to $150 \mu \mathrm{m}$ in diameter, as listed in Table 1. A36 mild steel was used as the substrate.

Two sets of experiments were performed to study the characteristics of powder flow. The first set of experiments was designed to capture the powder flow characteristics by image analysis. In the second set of experiments, the clads were deposited on the mild steel. The clad geometry and powder catchment efficiency were identified. The powder feeding and net clad weights were measured to determine the powder catchment efficiency under different process parameters.

\section{Mathematical Model of Gas-Powder Flow}

\section{Modeling Approach and Assumptions}

Gouesbet and Berlemont [9] made a comprehensive review of the turbulent two-phase modeling methods and found that the Eulerian-Lagrangian model was one of the most powerful tools that could be used to predict the dispersed particle behavior in the continuous turbulent flow. The gas flow phase calculation was based on the time-
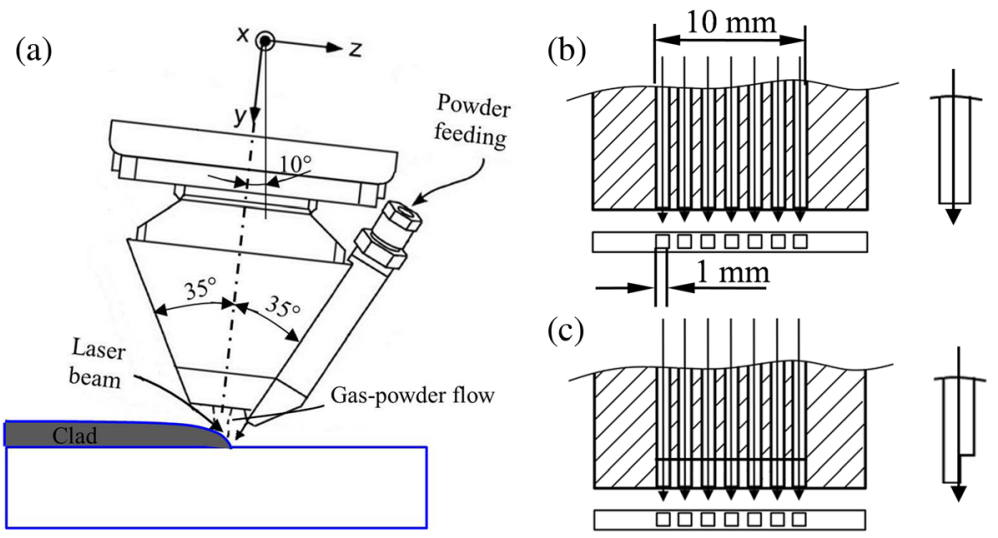

Fig. 2 (a) Configuration of the powder feeding nozzle with the laser head [22], and the exit geometry of the nozzle: (b) closed and (c) opened on one side 
averaged Navier-Stokes equations together with the k- $\varepsilon$ turbulence model, while the discrete phase calculation was based on the particle track model which predicts the particle trajectory by integrating the force balance on the particle. Two-way coupling between the two phases was calculated alternatively by solving the continuous and discrete equations. Several assumptions were made in the model: (1) the powder and the gas flow had the same inlet velocity, (2) the gravitational, drag and buoyant forces were included, and (3) the inter-particle collision was ignored.

\section{Modeling of Turbulent Flow}

A time-averaged turbulent gas model was set up to solve the governing equations expressed as $[19,20]$ :

Conservation of mass:

$$
\frac{\partial\left(\rho u_{i}\right)}{\partial x_{i}}=0
$$

where $u_{i}$ and $x_{i}$ represent the velocity and position.

Conservation of momentum:

$$
\frac{\partial}{\partial x_{i}}\left(\rho u_{i} u_{j}\right)+\frac{\partial}{\partial x_{i}}\left(\rho \overline{u_{i}^{\prime} u_{j}^{\prime}}\right)=-\frac{\partial p}{\partial x_{i}}+\frac{\partial}{\partial x_{j}}\left[\mu\left(\frac{\partial \overline{u_{i}}}{\partial x_{j}}+\frac{\partial \overline{u_{j}}}{\partial x_{i}}\right)\right]+\varnothing_{i}
$$

where $\mathrm{i}, \mathrm{j}=1,2,3, \rho$ is the density of gas, $u_{i}$ the velocity in the $i$-th direction, $\mathrm{p}$ is the pressure, $\left(\rho \overline{u_{i}^{\prime} u_{j}^{\prime}}\right)$ is the Reynolds stress, and $\varnothing_{i}$ is the source term that represents the coupled momentum transport from the particle phase [2]:

$$
\varnothing_{i}=\sum_{j=1}^{n_{C}} \frac{3 \mu C_{D} R e_{p}}{4 \rho_{p} D_{p}^{2}}\left(u_{p, i}-u_{i}\right) \dot{m}_{p, j} \Delta t_{j}
$$

is the particle mass rate for the $\mathrm{j}$-th trajectory passing through a cell, $\Delta t_{j}$ is the time that a particle on the j-th trajectory takes to pass through the cell, $n_{C}$ is the total number of particle trajectories passing through the cell, $\operatorname{Re}_{p}$ and $C_{D}$ are the Reynolds number and drag coefficient of a particle, and $\rho_{p}, D_{p}$ and $u_{p, i}$ are the density, diameter, and velocity in $\mathrm{i}$-th direction of a particle, respectively.

$\mu$ is the effective dynamic viscosity of the continuous phase:

$$
\mu=\mu_{l}+\mu_{t}
$$

$\mu_{l}$ is the laminar viscosity:

$$
\mu_{l}=\frac{\rho V_{\infty} l}{\operatorname{Re}}
$$


$\mu_{t}$ is the turbulent viscosity:

$$
\mu_{t}=\frac{C_{\mu} \rho k^{2}}{\varepsilon}
$$

\section{Modeling of Particle Phase}

The dispersed particles were treated as a continuous flow in the Lagrangian model and the kinematic change of powder was considered by calculating the particle-gas interaction and particle-wall collision. In FLUENT, the trajectory of each dispersed particle was predicted by integrating the force balance on the particle in a Lagrange coordinate system. The force balance can be written as [19]:

$$
\frac{d u_{p, i}}{d t}=\widehat{F_{D}}+\frac{g_{i}\left(\rho_{p}-\rho\right)}{\rho_{p}}+\widehat{F_{i}}
$$

where $\widehat{F_{i}}$ is the additional force acting on the particles and $\widehat{F_{D}}$ is the drag force, both on a per unit particle mass in the $i$-th direction.

$$
\begin{aligned}
\widehat{F_{D}} & =F_{D}\left(u_{i}-u_{p, i}\right) \\
F_{D} & =\frac{3 \mu C_{D} R e_{p}}{4 \rho_{p} D_{p}^{2}}
\end{aligned}
$$

where $R e_{p}$ is the particle Reynolds number defined by:

$$
R e_{p}=\frac{D_{p}\left|u_{i}-u_{p, i}\right|}{\mu}
$$

and $C_{D}$ is the drag coefficient. Generally, $C_{D}$ depends on the shape and conditions of the flow. For non-spherical particles, the drag coefficient $C_{D}$ was defined by Eq. (10) [10]:

$$
C_{D}=\frac{24}{R e_{p}}\left(1+b_{1} R e_{p}^{b_{2}}\right)+\frac{b_{3} R e_{p}}{b_{4}+R e_{p}}
$$

Where

$$
\begin{aligned}
b_{1} & =\quad \exp \left(2.3288-6.4581 \varphi+2.4486 \varphi^{2}\right) \\
b_{2} & =\quad 0.0964+0.5565 \quad \varphi \\
b_{3} & =\exp \left(4.905-13.9844 \varphi+18.4222 \varphi^{2}-10.2599 \varphi^{3}\right) \\
b_{4} & =\exp \left(1.4681+12.2584 \varphi-20.7322 \varphi^{2}+15.8855 \varphi^{3}\right)
\end{aligned}
$$

where $\varphi$ is surface factor:

$$
\varphi=\frac{S}{S}
$$


where $\mathrm{s}$ is the surface area of a sphere with the same volume of the particle and $\mathrm{S}$ is the actual surface area. The particle velocity can be acquired by:

$$
\frac{d x_{i}}{d t}=u_{p, i}
$$

Then a particle trajectory could be obtained by using Eqs. (7) and (13).

In FLUENT the particle size distribution could be described by a Rosin-Rammler function:

$$
Y_{d}=e^{-\left(D_{p} / \bar{D}_{p}\right)^{n}}
$$

where $\mathrm{Y}_{\mathrm{d}}$ is the mass fraction of the particles with a diameter greater than $\mathrm{D}_{\mathrm{p}}$, $\bar{D}_{p}$ is the mean diameter, and $\mathrm{n}$ is the spread parameter. According to the powder properties listed in Table $1, \bar{D}_{p}$ was calculated to be 95.36 and $\mathrm{n}$ was 3.14. The particle size distribution in weight is shown in Fig. 3. The column chart was plotted based on the powder sieve analysis in which each column represented the weight of the particles blocked by that mesh size. The black line was plotted using Rosin-Rammler function.

In order to accurately predict the powder concentration distribution, a non-uniform free mesh is adopted to the zones of high powder concentrations. Hexahedral finite elements are utilized in this analysis. The finest element size is set to $0.2 \mathrm{~mm}$ and a coarse mesh with element size up to $0.8 \mathrm{~mm}$ is used in the domain far from the powder flow.

The powder catchment efficiency in the simulation is counted by the ratio of the mass of particles which were trapped by the molten pool area to the total fed particles. The molten pool size is defined to be $12 \times 5 \mathrm{~mm}$ measured from the CCD image shown in Fig. 4.

\section{Results and Discussion}

\section{Powder Flow Structure}

Figure 5a-b depict the powder stream structures formed by the nozzles with the closed and open exit, respectively. The front views of the powder flow in simulation and experiment look similar at the closed and open exits, while the side view of the powder flow in simulation and experiment shows that the powder flow expanded more at the closed exit due to the opening at the nozzle exit releasing the gas pressure. Consequently, the velocity of the particles was slow down and the trajectory of the particles

Table 1 Size distribution of Fe-based alloy powder

\begin{tabular}{llllllll}
\hline Powder size $(\mu \mathrm{m})$ & $>45$ & $45 \sim 53$ & $53 \sim 75$ & $75 \sim 106$ & $106 \sim 150$ & $150 \sim 180$ & $>180$ \\
\hline Mass fraction, \% & 1.11 & 4.56 & 28 & 44.96 & 20.6 & 0.77 & 0 \\
\hline
\end{tabular}




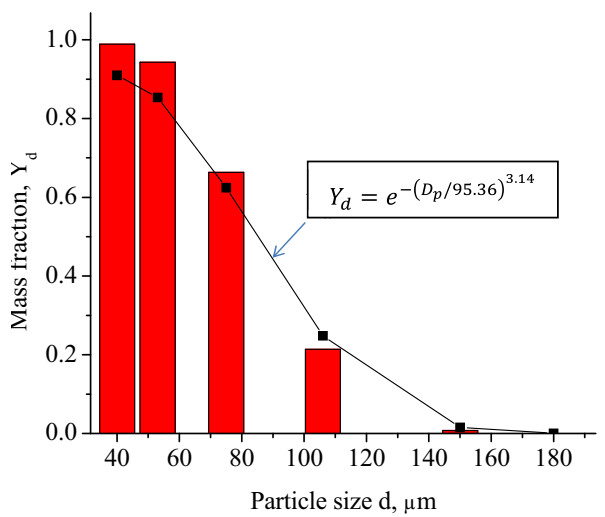

Fig. 3 Comparison between measured and Rosin-Rammler particle size distribution

dispersed in the gas was changed. The opening at one side of the nozzle exit was beneficial for reducing the divergence of the powder. In the following study, the nozzle with an open exit was selected.

The velocity of the powder particles was an important variable that influenced the powder flow characteristics. At a powder feeding rate $(\mathrm{m})$ of $50 \mathrm{~g} / \mathrm{min}$ and a carrier-gas flow rate $(\mathrm{CG})$ of $15 \mathrm{SCFH}\left(0.425 \mathrm{~m}^{3} / \mathrm{h}\right)$, Fig. 6 represents the gas and particle velocity distribution. The inlet velocity of the gas $v_{g}$ and particles $v_{p}$ were assumed to be the same and set to be $9.4 \mathrm{~m} / \mathrm{s}\left(V_{g}=V_{p}=4 \frac{(C G)}{\left.\pi d_{i}^{2}\right)}\right.$, where $\mathrm{d}_{\mathrm{i}}$ is the diameter of the inlet, which is $4 \mathrm{~mm}$. Due to the features of the structure inside the powder nozzle, the gas flow carrying the particles tended to exit from the side channels. It was predicted that the velocity of gas near the exit was in the range of $6 \sim 10 \mathrm{~m} / \mathrm{s}$, while the velocity of the particles was in the range of $2.8 \sim 4.5 \mathrm{~m} / \mathrm{s}$. These results indicated that the dynamic

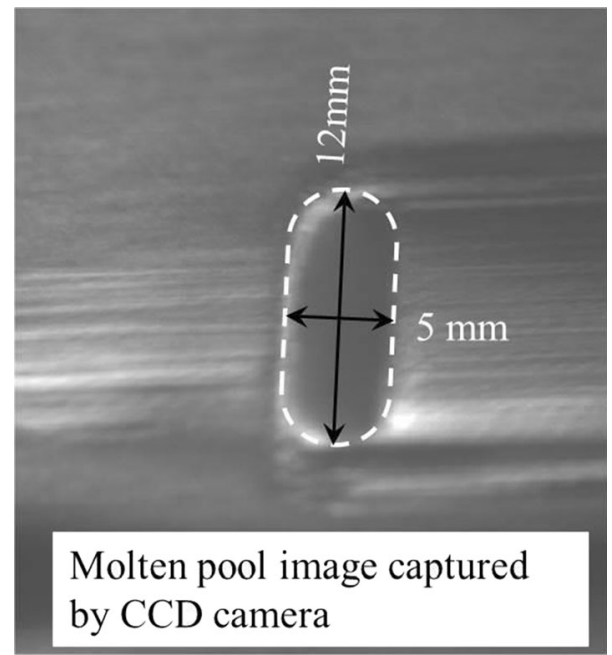

Fig. 4 Image of molten pool 
(a) Simulation

Experimental
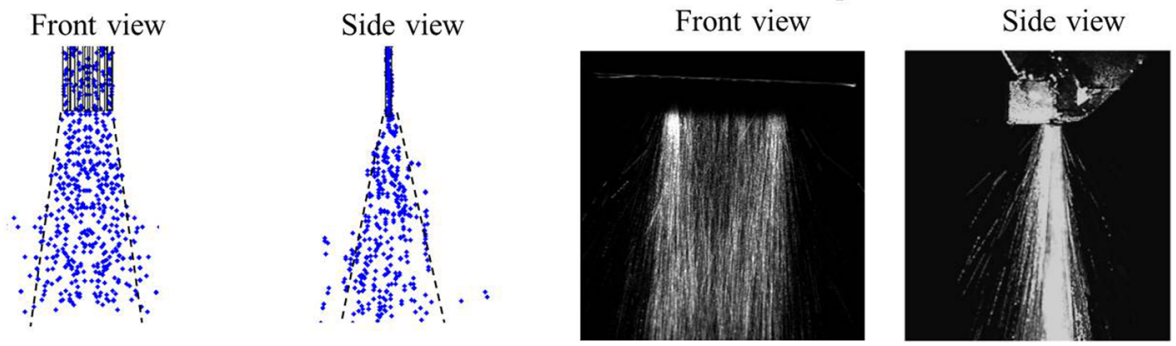

(b)

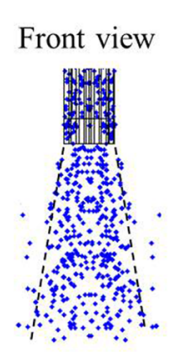

Simulation
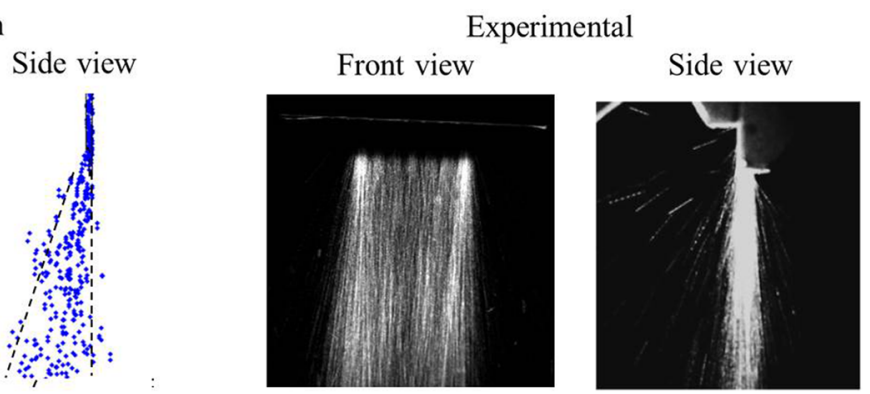

Fig. 5 The particle track after injection from the nozzle with (a) a closed exit and (b) an open exit

behavior of the particles was closely related to the gas field, whereas replacing the powder velocity with the gas velocity was not accurate for the quantitative analysis. The numerical model was verified by the experimental measurements with respect to the powder particle velocity. In the imaging technique, the particle velocity was measured based on the trace length of the particle captured by a CCD camera at an exposure time of $0.2 \mathrm{~ms}$, as shown in Fig. 7. The particle velocity, $V_{\mathrm{p}}$, could be expressed in terms of the trace length of the particle $(l)$ divided by the exposure time $(t)$ as:

$$
V_{p}=\frac{l}{t}
$$

Lengths of the traces of particles near the nozzle exit were measured. The measured results matched very well with the predicted velocity of the particles.

Figure 8 presents the powder concentration distribution from the front view and the cross-sections at several distances from the nozzle tip. The powder concentration near the edges of the nozzle was higher than at the center, which related to the characteristics of the gas field as described above. The powder was pushed out of the nozzle from the row of seven channels with a dimension of $10 \times 1 \mathrm{~mm}$. At the distance of $20 \mathrm{~mm}$ from the nozzle exit the powder stream expanded to $14 \times 4 \mathrm{~mm}$. Given that the powder stream began to diverge after exiting the nozzle, the deposition distance should have been as small as possible in order to achieve the highest powder catchment efficiency. However, due to the heating of the nozzle tip caused by the large reflection of laser beam, the powder nozzle 
(a)

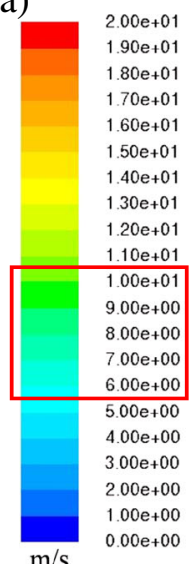

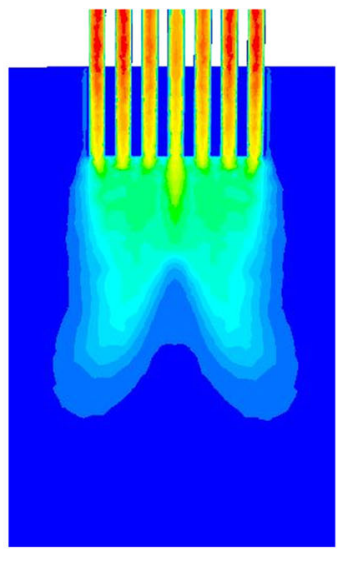

(b)

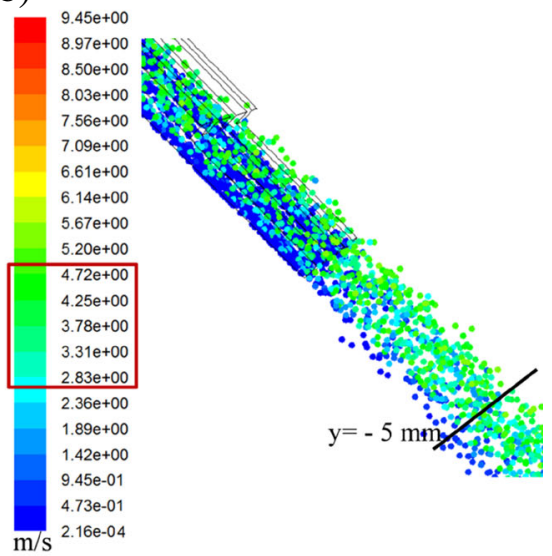

Fig. 6 Velocity field of (a) the carrier-gas flow and (b) the powder flow

was easily clogged by the ricochet of powder particles. Since the laser spot size was $12 \times$ $3 \mathrm{~mm}$, the optimal position of intersection between laser beam and powder flow was determined to be at a distance of 8 to $12 \mathrm{~mm}$ from the nozzle tip.

The powder flow image captured by the CCD camera is shown in Fig. 9a with a color legend in which red indicated a high powder concentration and blue indicated a diluted powder concentration. Similar distributions of powder concentration were found from numerical results. Figure $9 \mathrm{~b}$ shows the cross section of the clad deposited at the distance of $10 \mathrm{~mm}$ with a laser power of $P=3.5 \mathrm{~kW}$. The concave surface profile of the clad was associated with the surface tension of the molten pool and the

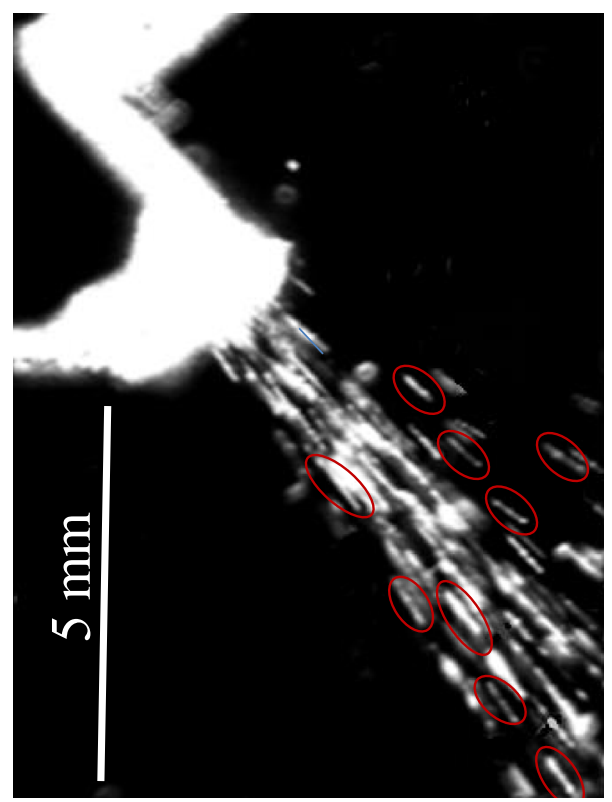

Fig. 7 Particle track image corresponding to model verification case 


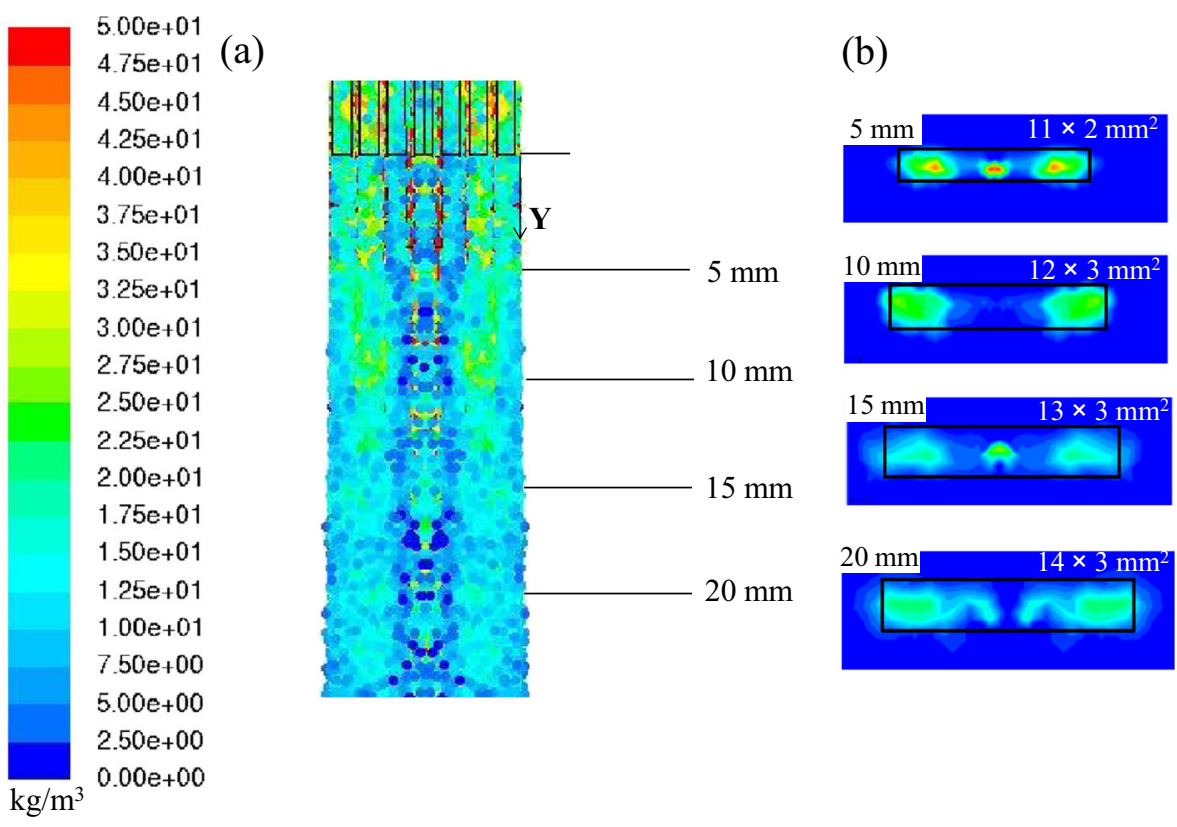

Fig. 8 Powder concentration distribution of single nozzle at (a) the front view and (b) cross-sectional view at several distances from the nozzle tip

distribution of the feeding material. One method to obtain a flat clad surface could be performed via optimization of the carrier gas flow rate. Increasing the carrier-gas flow rate could improve the full mixing of the powder inside the nozzle and mitigate the difference of the powder concentration over the width of the nozzle after exiting the nozzle. Figure 10 shows the powder flow image and the resulted clad when the carriergas flow rate was increased to $20 \mathrm{SCFH}$. The clad surface became flatter but its height was decreased, which indicats that the powder catchment efficiency was decreased at a high carrier-gas flow rate.

\section{Effects of Particle Properties on the Powder Feeding Behavior}

The powder properties (particle size, density, and shape) largely influence the spatial distribution of the particles [16]. In order to investigate the effects of individual factor like particle size, density, and shape, each factor was studied independently from each in the simulation. In practical application, the powder properties are more dispersive. Numerical analysis would provide guidance for analyzing the experimental results and give a better understanding for the efficient use of powder.

\section{Effects of Particle Size on the Powder Feeding Behavior}

In order to study the effects of particle size, the size of Fe-based particles was discretized to be $10,50,100,200$, and $300 \mu \mathrm{m}$, respectively. The particle velocity was decreased with an increase in the particle size, as shown in Fig. 11. With a constant inlet velocity of $9.4 \mathrm{~m} / \mathrm{s}(\mathrm{CG}=15 \mathrm{SCFH})$, the particles with the size of $10 \mu \mathrm{m}$ reached 
(a)
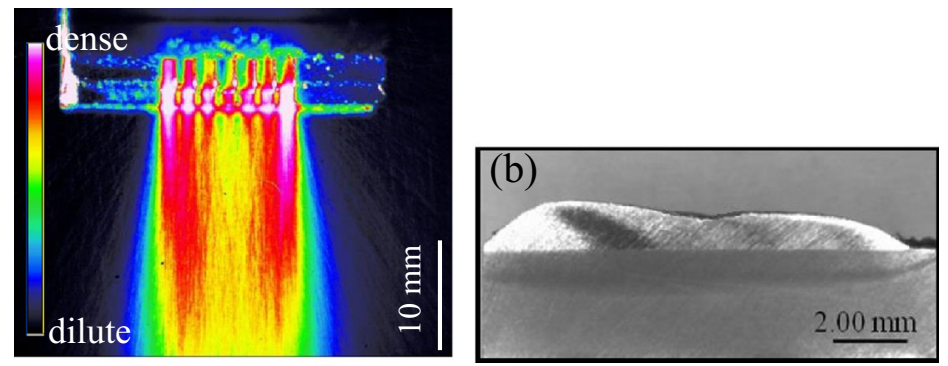

Fig. 9 (a) powder flow image captured by CCD camera (b) cross-section of the deposited clad produced at a distance of $10 \mathrm{~mm}$ between the outlet of the nozzle and the molten pool $(\mathrm{m}=50 \mathrm{~g} / \mathrm{min}$ and CG=15 SCFH)

the averaged velocity of $9.24 \mathrm{~m} / \mathrm{s}$ at the exit of the nozzle, whereas the averaged velocity of the particles with the size of $300 \mu \mathrm{m}$ was of $1.25 \mathrm{~m} / \mathrm{s}$. The particle velocity represented the combined effects of the drag force by the carrier gas, the gravity of the particle and the collision with the walls. The restitution coefficients of the particles were mainly affected by the particle shape and the impact angle [21]. Here, a constant restitution coefficient of 0.5 was selected [15]. From Eq. (7), it was found that the variation of the particle size had a dominant effect on the drag force, which was in an inverse-proportional relationship. The smaller the particle, the larger the drag force is.

Figure 12 shows the streamline plot of the carrier gas. The single nozzle was angled $45^{\circ}$ such that the carrier gas impact the back of the molten pool. The particles at track 2 would be trapped by the molten pool, while particles at track 1 and 3 would be reflected by the solid substrate. Figure 13a-c represent the particle tracks of some selected particle size after ejected out of the nozzle. It can be observed that the fine particles were more vulnerable to the influences of the turbulent gas flow. A few particles were suspended above the molten pool. Most of the fine particles following closely to the main gas stream were carried to the front of the molten pool along track 1 and reflected. With an increase in particle size, the particles were more dispersed after collision with the nozzle walls. In addition, because of the effect of gravity, more particles tended to move along tracks 2 and 3. Most of the particles with the size of $100 \mu \mathrm{m}$ entered the molten pool along track 2 . Remaining particles were reflected away from the surface. The particles with the size of $300 \mu \mathrm{m}$ slid down against the wall of the nozzle slowly. Most of them travelled along the track 3 and reached the front of the molten pool. Large-sizedparticles that were not trapped by the molten pool remained on the substrate surface. Figure 13d shows their corresponding powder concentration distribution at a

(a)
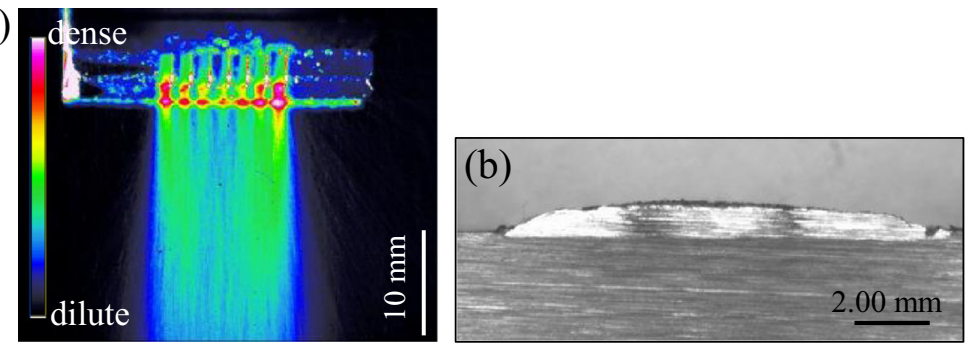

Fig. 10 (a) powder flow image captured by CCD camera (b) cross-section of the deposited clad produced at a distance of $10 \mathrm{~mm}$ between the outlet of the nozzle and the molten pool $(\mathrm{m}=50 \mathrm{~g} / \mathrm{min}$ and $\mathrm{CG}=20 \mathrm{SCFH})$ 


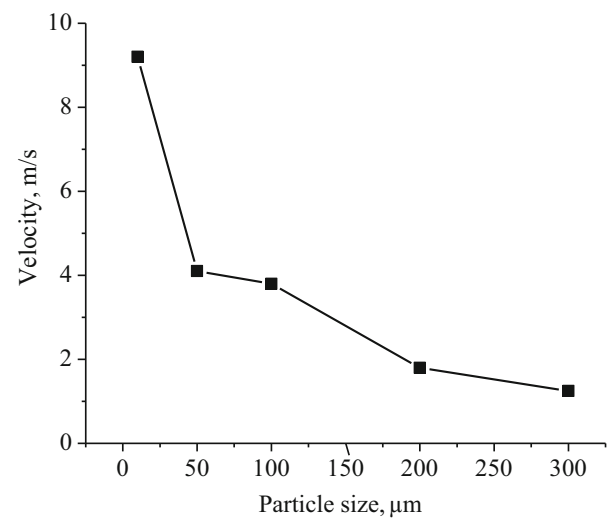

Fig. 11 Particle velocity versus particle size

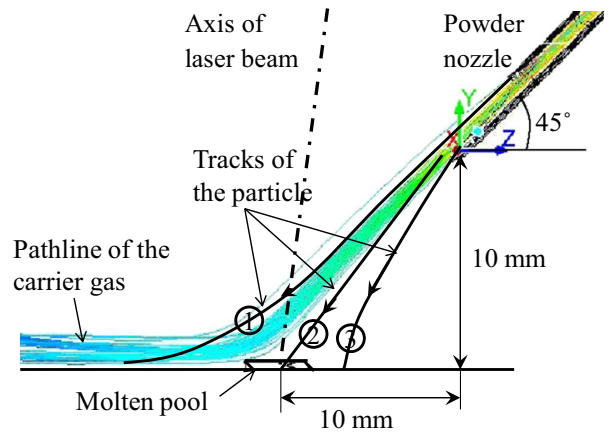

Fig. 12 Streamline plot of the carrier gas

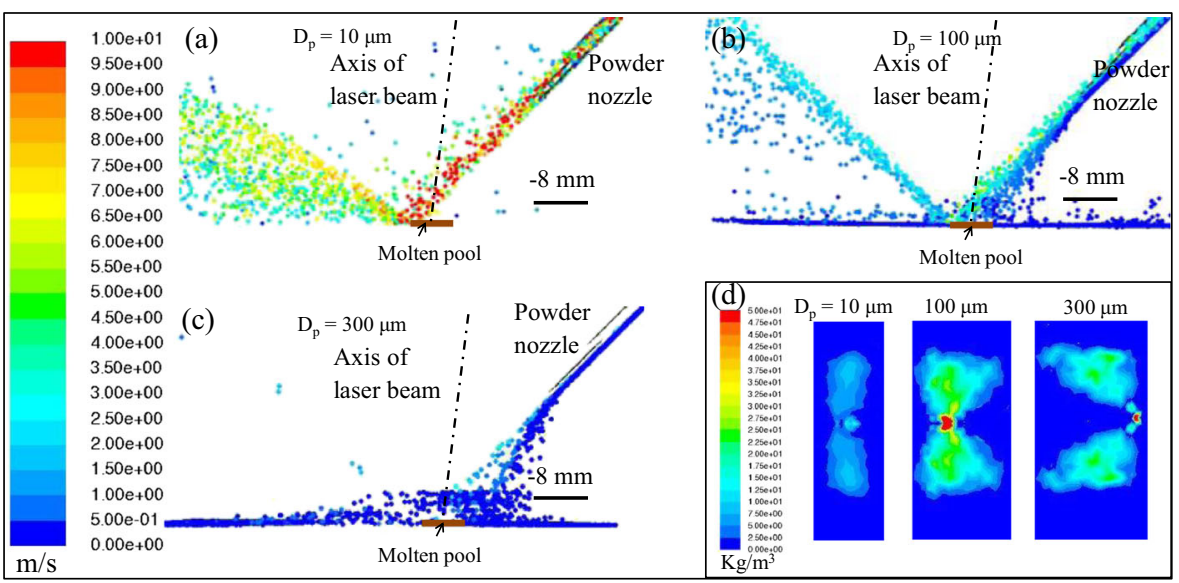

Fig. 13 Particle track at different particle sizes of (a) $10 \mu \mathrm{m}$, (b) $100 \mu \mathrm{m}$ and (c) $300 \mu \mathrm{m}$ and powder concentration (d) at the plane of $Y=-8 \mathrm{~mm}$ 
distance of $8 \mathrm{~mm}$ from the nozzle tip. For the small particles that followed the gas flow more closely, the powder concentration was low and their distribution was more uniform below the exit of the nozzle. For the particles larger than $100 \mu \mathrm{m}$, the effects of gravity and inertia were more pronounced, resulting in a distinct separation of the powder flow after expulsion from the nozzle.

For the powder flow comprised of Fe-based powder with particle sizes ranging from 45 to $180 \mu \mathrm{m}$, it can be inferred that the small particles tended to travel at the upside of the powder flow with high velocity, while the large particles tended to travel at the downside of the powder flow with low velocity. Moreover, particles were more concentrated near the side than the center over the nozzle width. The particles with the sizes around $100 \mu \mathrm{m}$ were the main contributor to the powder catchment efficiency, as shown in Fig. 14.

\section{The Effects of Particle Density on the Powder Feeding Behavior}

Three types of particles with constant particle sizes of $100 \mu \mathrm{m}$ were ejected out of the powder nozzle. The density of Fe, Ni and WC was 8030,8900 and $15,600 \mathrm{~kg} / \mathrm{m}^{3}$, respectively. From Eq. (7) it is clear that the force acting on the particle is proportional to the gravity while inversely proportional to the drag force. Hence, the larger the particle density, the more pronounced the effect of gravity. Figure 15 shows that the particle velocity decreased with an increase in the particle density.

Figure 16a-c shows the particle tracks and Fig. 16d shows the powder concentration distribution at different densities. With an increase in the particle density, the particles were more dispersed. An amount of the particles that were not trapped by the molten pool bounced off the substrate back into the air over the molten pool. This would increase the attenuation of the laser beam by the powder particles and decrease the powder catchment efficiency. It can be inferred that in the matrix metal composite composed of the particles with different densities, the particles with the density close to WC would be lost more, as shown in Fig. 17. Moreover, due to the difference in the other physical properties of these particles (Fe, Ni, WC) would dramatically change the clad properties. Anandkumar et al. [1] found that the particle velocity had a large influence on the microstructure of the clad in the Al-Si metal matrix composite

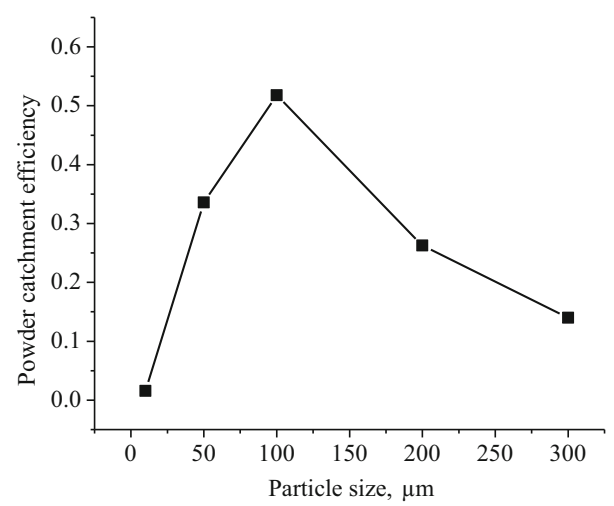

Fig. 14 Particle catchment efficiency versus particle size 


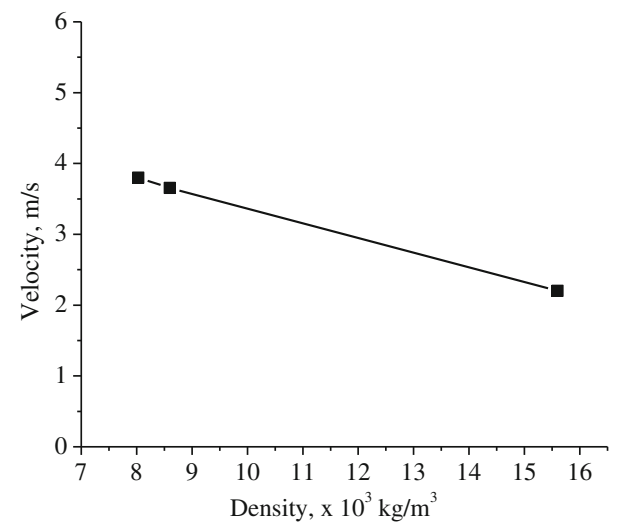

Fig. 15 Particle velocity versus particle density $\left(D_{p}=100 \mu \mathrm{m}\right)$

reinforced with SiC. This work was helpful for better understanding of the mechanism of the mass and heat transfer in the deposition and for building a base to optimize the microstructure of metal matrix composites.

\section{Effects of Particle Shape on the Powder Feeding Behavior}

In the production of the powder, particles were usually not purely spherical, as shown in Fig. 18. Here the irregularity of the particle shape was described by the concept of surface factor (Eq. 12), which for the non-spherical particle was less than 1. The particle shape had a significant effect on the interaction between the solid particle and the gas field. Previous work had demonstrated that the particle shape mainly affected the drag coefficient [4] and the smooth powder delivery [7]. From Eq. (10), it was confirmed that the non-spherical particle had a much higher drag coefficient, resulting in a higher

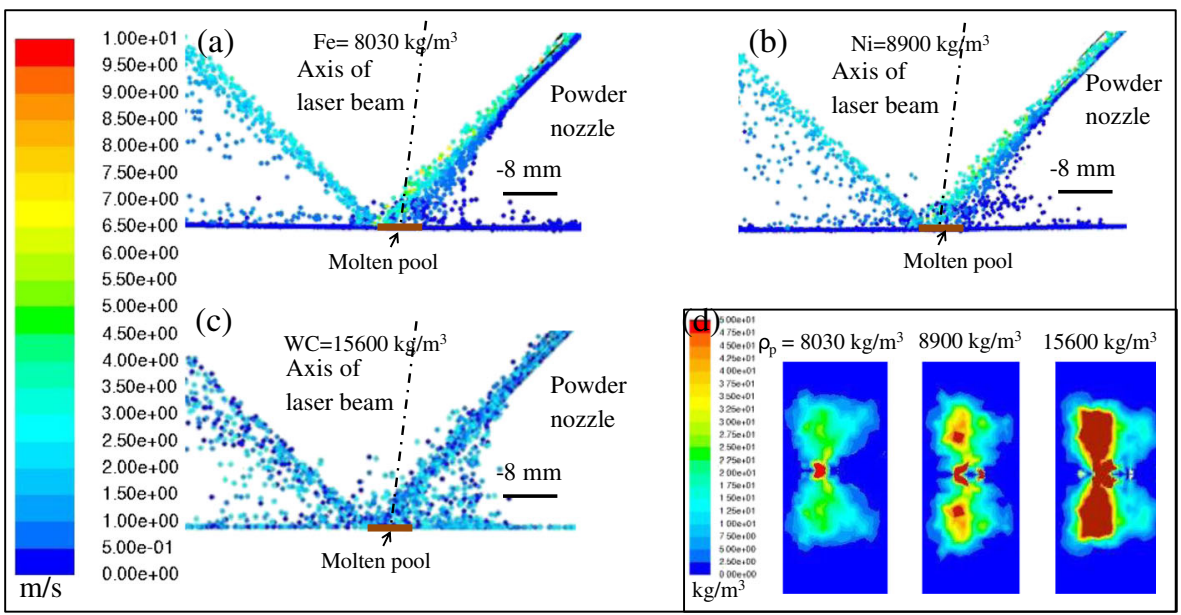

Fig. 16 Particle tracks of different particle densities of (a) $8030 \mathrm{~kg} / \mathrm{m}^{3}$, (b) $8900 \mathrm{~kg} / \mathrm{m}^{3}$ and (c) $15,600 \mathrm{~kg} / \mathrm{m}^{3}$ and their powder concentration (d) at the plane of $Y=-8 \mathrm{~mm}$ 


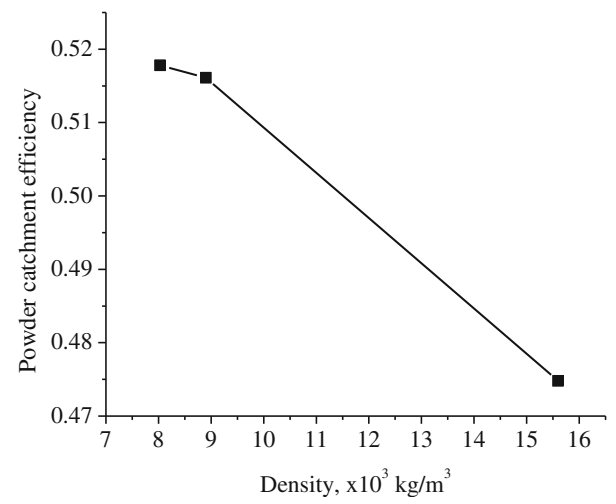

Fig. 17 Powder catchment efficiency versus particle density
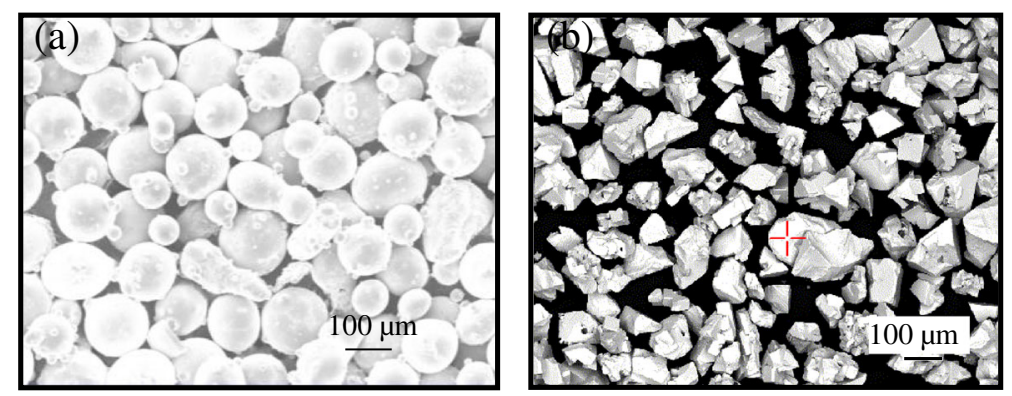

Fig. 18 Powder particle morphology of (a) spherical, and (b) angular

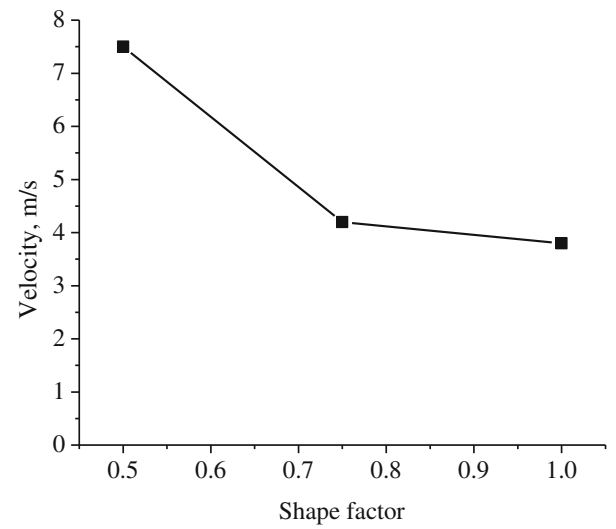

Fig. 19 Particle velocity versus particle surface factor 


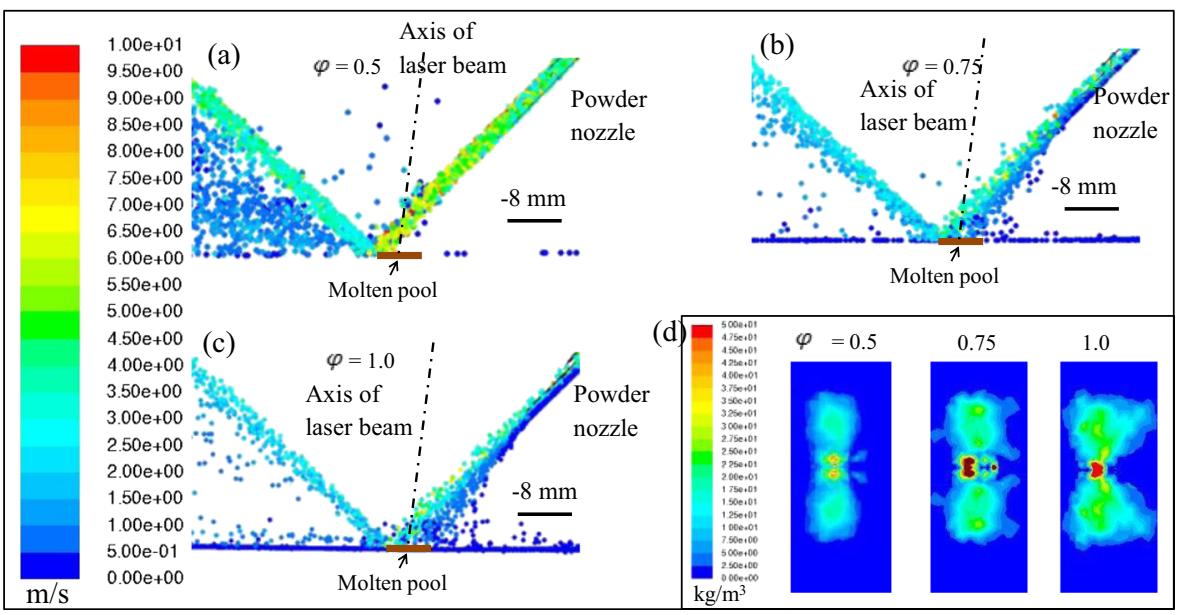

Fig. 20 Particle tracks of Fe with different surface factors (a) 0.5 , (b) 0.75 and (c) 1.0 and their powder concentration (d) at the plane of $Y=-8 \mathrm{~mm}$

velocity, as shown in Fig. 19. The tracks of the particles and the powder concentration for different shape factors are shown in Fig. 20. Due to the angular shape of the nonspherical particles, the powder flow was not as free-flowing as the spherical particles. Moreover, due to the collision between the wall and particles, the directions of the nonspherical particles at the exit of the nozzle are more scattered. Some particles escaped from the main powder stream at the exit of the nozzle. For the remaining particles, they impact the substrate in a narrower zone, but the total powder catchment efficiency was low, as shown in Fig. 21. It can be inferred that for the powder mixed with different particle shapes, the spherical particles made the main contribution for the powder catchment efficiency.

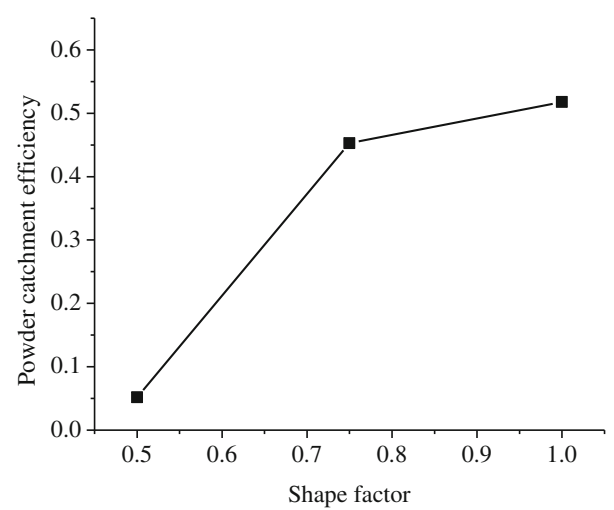

Fig. 21 Powder catchment efficiency versus surface factor 

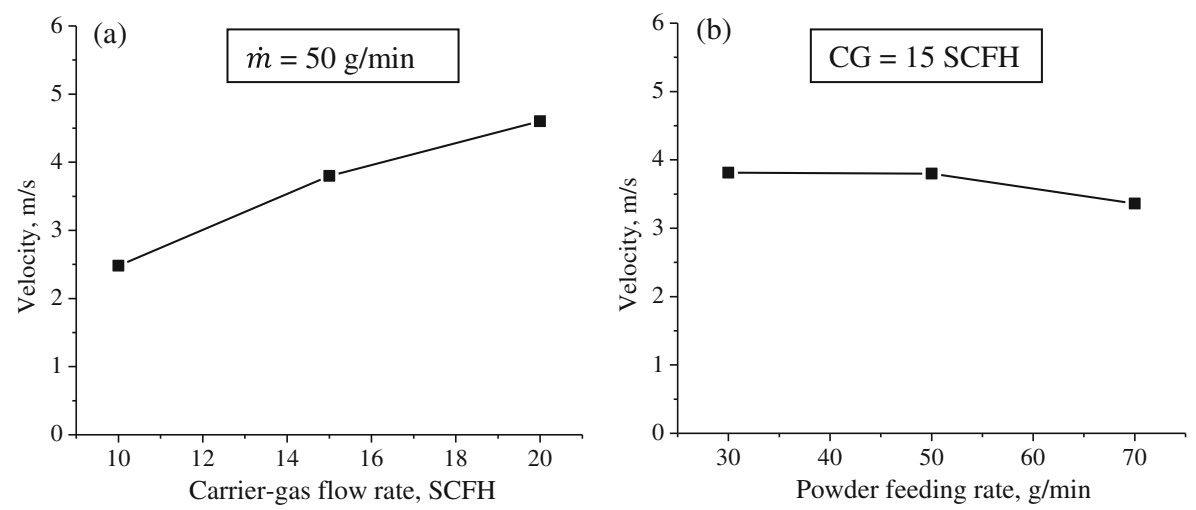

Fig. 22 Particle velocity versus (a) carrier-gas flow rate and (b) powder feeding rate

\section{Effects of the Powder Feeding Parameters on the Powder Feeding Behavior}

In the investigation of the effects of the powder feeding parameters on the powder flow characteristics, the feeding material of Fe-based alloy with a surface factor of 1.0 is simulated. The effect of the carrier-gas flow rate and the powder feeding rate on particle velocity is shown in Fig. 22. The averaged particle velocity was increased from 2.5 to $4.9 \mathrm{~m} / \mathrm{s}$ when the carrier-gas flow rate was increased from 10 to $20 \mathrm{SCFH}$. The average particle velocity was almost constant with the variation of the powder feeding rate. That implied that the inter-particle collision had little effect on the motion of the particles due to a very low volume fraction of the particles.

Figure 23 presents that the powder catchment efficiency decreased with an increase in powder feeding rate. The powder catchment efficiency decreased with an increase in carrier-gas flow rate at $m=30 \mathrm{~g} / \mathrm{min}$ and $50 \mathrm{~g} / \mathrm{min}$. However, at $m=70 \mathrm{~g} / \mathrm{min}$, the powder catchment efficiency was very low at $\mathrm{CG}=10 \mathrm{SCFH}$. The low efficiency was

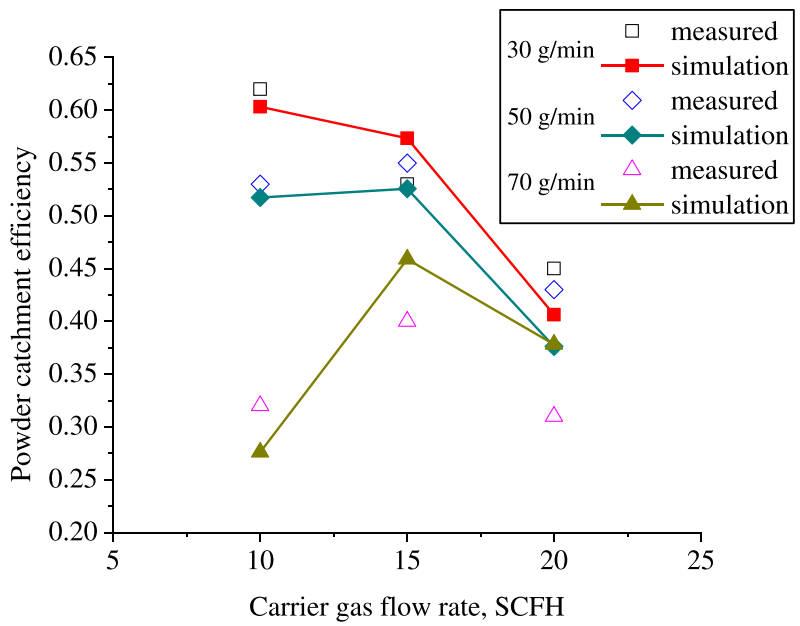

Fig. 23 Powder catchment efficiency at different powder feeding parameters 
caused by the nozzle clogging. The kinetic energy of the gas field at a low flow rate was not high enough to press such an amount of mass out of the nozzle and ultimately affected the powder delivery, and was thus not accurately predicted by simulation under the same conditions.

\section{Conclusions}

Several powder delivery issues associated with the HPDDL cladding process were studied by both numerical and experimental methods. The conclusions were drawn as follows:

(1) The exit shape of the nozzle affected the powder flow structure. The opening at one side of the nozzle exit controlled the pressure distribution and was beneficial in providing more coherent powder flow.

(2) The powder concentration distribution over the nozzle width was not uniform and affected the deposited clad geometry. The powder concentration at the side region of the nozzle was higher than at the central region. A clad with a flat surface could be obtained by adjusting the carrier-gas flow rate which will provide more uniform powder distribution across the outlet of the nozzle.

(3) The particle properties including particle size, density, and shape factor and the powder feeing parameters including powder feeding rate, and carrier-gas flow rate had effects on the particle velocity, its trajectory, powder concentration and catchment efficiency.

(4) It was found that higher particle velocities would be observed with fine particles, while the larger particles were more dispersed and tended to be separated into two powder streams near the side region of the nozzle. With the increase of particle density, the particle velocity decreased. Non-spherical particles had higher velocities and were more vulnerable to the effect of the turbulent gas field.

(5) The particle velocity was increased with an increase in carrier-gas flow rate, while nearly unaffected by the powder feeding rate. The powder catchment efficiency was increased with a decrease in carrier-gas flow rate. However, the carrier-gas flow rate needed tostrong enough to push the particles out of the powder nozzle.

Acknowledgments The author would like to thank Andrew Socha, Dr. Fanrong Kong, Dr. Prabu Balu research engineers in the Center of Laser-aided Manufacturing for the help in the execution of experiments and Dr. Bruno Acklin Director of Engineering of Coherent for the instruction in the completion of this work. This work was funded by the NSF Grant No. IIP-1034652.

\section{References}

1. Anandkumar, R., Almeida, A., Vilar, R., Ocelik, V., De Hosson, J.T.M.: Influence of powder particle injection velocity on the microstructure of $\mathrm{Al}-12 \mathrm{Si} / \mathrm{SiC}_{\mathrm{p}}$ coatings produced by laser cladding. Surf. Coat. Technol. 204, 285-290 (2009) 
2. Babets, K.: Numerical modeling and optimization of waterjet based surface decontamination. Ph. D. Thesis, The New Jersey Institute of Technology. (2001)

3. Balu, P., Leggett, P., Kovacevic, R.: Parametric study on a coaxial multi-material powder flow in laserbased powder deposition process. J. Mater. Process. Technol. 212, 1598-1610 (2012)

4. Black, D.L., McQuay, M.Q.: Laser-based particle measurements of spherical and nonspherical particles. Int. J. Multiphase Flow 27, 1333-1362 (2001)

5. Cook, C.M., Haake, J.M., Zediker, M.S., Banaskavich, J.M.: Diode laser cladding produces high quality coatings. International SAMPE Technical Conference, Baltimore, 32, 910-921 (2000)

6. Doubenskaia, M., Pavlov, M., Grigoriev, S., Smurov, I.: Definition of brightness temperature and restoration of true temperature in laser cladding using infrared camera. Surf. Coat. Technol. 220, 244 247 (2013)

7. Gao, L.J., Yan, Y., Lu, G., Carter, R.M.: On-line measurement of particle size and shape distributions of pneumatically conveyed particles through multi-wavelength based digital imaging. Flow Meas. Instrum. 27, 20-28 (2012)

8. Gong, X.Y., Zhang, Y.Z., Liu, M.K.: Powder transport model for laser cladding by lateral powder feeding: I. powder flow field with cylindrical distribution. Int. J. Adv. Manuf. 67, 2501-2509 (2013)

9. Gouesbet, G., Berlemont, A.: Eulerian and Lagrangian approaches for predicting the behavior of discrete particles in turbulent flows. Prog. Energy Combust. Sci. 25, 133-159 (1999)

10. Haider, A., Levenspiel, O.: Drag coefficient and terminal velocity of spherical and nonspherical particles. Powder Technol. 58, 63-70 (1989)

11. Hu, D., Kovacevic, R.: Modelling and easuring the thermal behavior of the molten pool in closed-loop controlled laser-based additive manufacturing. Proc. Inst. Mech. Eng. B J. Eng. Manuf. 217, 441-452 (2003)

12. Li, L.: The advances and characteristics of high-power diode laser materials processing. Opt. Lasers Eng. 34, 231-253 (2000)

13. Lin, J., Steen, W.M.: Powder flow and catchment during coaxial laser cladding. Proc. SPIE 3097, 517528 (1997)

14. Meriaudeau, F., Truchetet, F., Dumont, C., Renier, E., Bolland, P.: Acquisition and image processing system able to optimization laser cladding process. Proc. ICSP 96, 1628-1631 (1996)

15. Pan, H., Liou, F.: Numerical simulation of metallic powder flow in a coaxial nozzle for the laser aided deposition process. J. Mater. Process. Technol. 168, 230-244 (2005)

16. Petrak, D., Rauh, H.: Optical probe for the in-line determination of particle shape, size, and velocity. Part. Sci. Technol. Int. J. 24, 381-394 (2006)

17. Pinkerton, A J., Li, L.: A verified model of the behavior of the axial powder stream concentration from a coaxial laser cladding nozzle. Proceeding of the Laser Materials Processing Conference (ICALEO), Scottsdale (2002)

18. Pinkerton, A.J., Li, L.: Modeling powder concentration distribution from a coaxial deposition nozzle for laser-based rapid tooling. J. Manuf. Sci. Eng. Trans. ASME 126, 33-41 (2004)

19. FLUENT 6.3 User Manual: Fluent Inc., Canonsburg, Pennsylvania, USA (2006)

20. Rajaratnam, N.: Turbulent Jets. Elsevier, Amsterdam (1976)

21. Sommerfield, M.: Modeling of particle-wall collisions in confined gas-particle flows. Int. J. Multiphase Flow 18, 905-926 (1992)

22. Washko, J.F., Parker, H.K., Brookshier, S.W.: Powder-delivery apparatus for laser-cladding, Patent Application: US 2012/0199564 A1 (2012)

23. Wen, S.Y., Shin, Y.C., Murthy, J.Y., Sojka, P.E.: Modeling of coaxial powder flow for the laser direct deposition process. Int. J. Heat Mass Transf. 52, 5867-5877 (2009)

24. Yang, N.: Concentration model based on movement model of powder flow in coaxial laser cladding. Opt. Lasers Technol. 41, 94-98 (2009)

25. Zekovic, S., Dwivedi, R., Kovacevic, R.: Numerical simulation and experimental investigation of gaspowder flow from radically symmetrical nozzles in laser-based direct metal deposition. Int. J. Mach. Tools. Manuf. 47, 112-123 (2007) 